\title{
Crosstalk between maternal perinatal obesity and offspring dopaminergic circuitry
}

\author{
Yuki Yasumoto and Tamas L. Horvath \\ Program in Integrative Cell Signaling and Neurobiology of Metabolism, Department of Comparative Medicine, Yale School of Medicine, New Haven, Connecticut, USA.
}

\begin{abstract}
The mechanism by which maternal obesity influences fetal brain development and behavior is not well understood. In this issue of the JCI, Lippert et al. showed that feeding maternal mice a high-fat diet (HFD) during lactation attenuated the activity of dopamine (DA) midbrain neurons and altered the DA-related behavioral phenotype seen in the offspring. The authors further suggested that the altered excitatory and inhibitory balance between D1 medium spiny neurons (MSN) and D2 MSN mediates this behavioral phenotype. These mechanisms may provide strategies for preventing the negative effects of maternal obesity on offspring development and adult health.
\end{abstract}

\section{Maternal environment affects brain development and mental health}

The idea that environment during early life affects health and wellbeing later in life is now widely recognized as the concept known as developmental origins of health and disease (DoHAD). Prenatal obesity has long-lasting consequences on the health of offspring (1). In addition, both human and rodent studies have shown that maternal obesity is associated with neurodevelopmental and psychiatric disorders in offspring, including attention-deficit disorder, cognitive delay, and autism $(2,3)$.

The main region of the brain that regulates energy balance is the hypothalamus. Previous work showed that maternal obesity and maternal high-fat diet (HFD) consumption impairs the hypothalamic melanocortin circuitry in mice (4). However, feeding behavior is also modulated by cognitive, appetitive, and emotional drives. In particular, dopamine (DA) within the mesocorticolimbic DA (MCL-DA) system regulates feeding as well (5). Dopaminergic neurons in the ventral tegmental area (VTA) and substantial nigra (SN) pars compacta are a major source of DA in the dorsal and ventral striatum, where DA binds to receptors located on striatal medium spiny neurons (MSNs). Notably, foods rich in sugar and fat stimulate DA release in the nucleus accumbens (NAc) that projects from the VTA (6). In the developing brain, inflammation caused by maternal obesity impairs the DA circuit and increases the risk for developing mental health disorders in offspring $(7,8)$. Thus, it is a reasonable hypothesis that maternal nutritional status may have a great influence on DA circuitry and function as well as behavior of the offspring.

In this issue of the JCI, Lippert et al. (9) used a time-restricted dietary manipulation during lactation in mice (4). They chose this model because the lactation period in rodents corresponds to the third trimester of pregnancy in humans, in which neurogenesis, synaptogenesis, and myelination within limbic structures occur $(10,11)$.

Delated Article: p.3761

Conflict of interest: The authors have declared that no conflict of interest exists.

Copyright: (c) 2020, American Society for Clinical Investigation.

Reference information: J Clin Invest. 2020;130(7):3416-3418. https://doi.org/10.1172/JCl138123.

How does maternal HFD feeding alter dopaminergic circuitry?

First, Lippert et al. (9) dissected the VTA and $\mathrm{SN}$ tissue from adult offspring and performed mRNA sequencing to examine changes in the dopaminergic system. Gene Ontology (GO) analysis and subsequent GO term clustering of the overlapping transcriptional changes between the VTA and SN revealed that maternal HFD altered gene expression related to neuronal development, ion channel expression, and locomotor behavior.

Next, the researchers investigated the morphology of the SN dopaminergic neurons by immunohistochemistry. They found impaired DA neuronal projections in the SN pars reticulata and the NAc without major alterations in DA neuron numbers in the offspring of mothers exposed to HFD during lactation. To study the firing properties of DA neurons in the SN, they performed perforated patch-clamp experiments. A smaller population of pacemaking neurons and a larger population of silent neurons were found in those mice. In addition, DA release in the NAc, which was evoked by electrical and chemogenic (DREADD) stimulation of the midbrain, was reduced (9).

These altered responses in the DA neurons resulted in DA-related behavioral changes in offspring of mothers exposed to HFD during lactation. While the aforementioned results were observed in both males and females, behavioral experiments revealed a distinct sexual dimorphism associated with elevations in DA-related behaviors, i.e., increased locomotion in males and increased intake of palatable food and sucrose in females (9). This sex difference in the DA-related behavioral phenotype is consistent with human data in that a male dominance is common in ADHD and a female dominance is common with overweight or obesity (12).

DA receptors (DRs) are G-coupled receptors associated with the $G_{s \alpha / \text { olf }}$ protein for the D1 family (DRD1) and with the Gi protein for the D2 family (DRD2) 
A Control

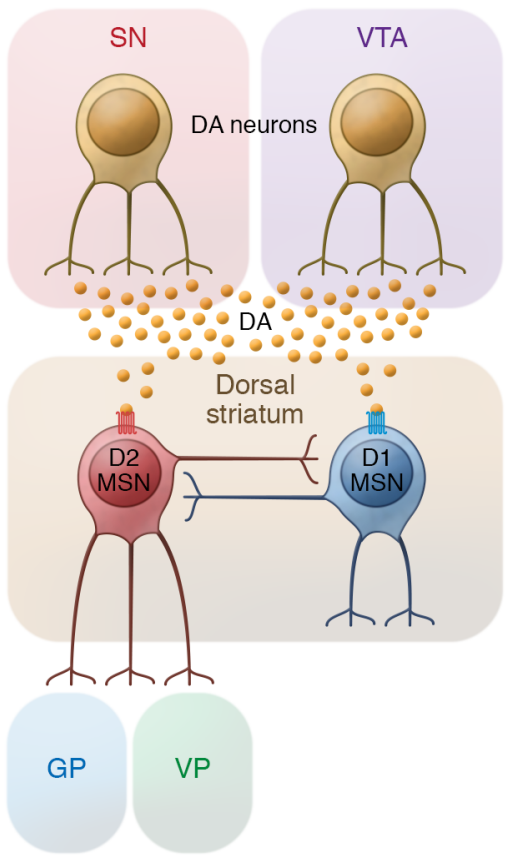

B Offspring of mothers exposed to HFD during lactation

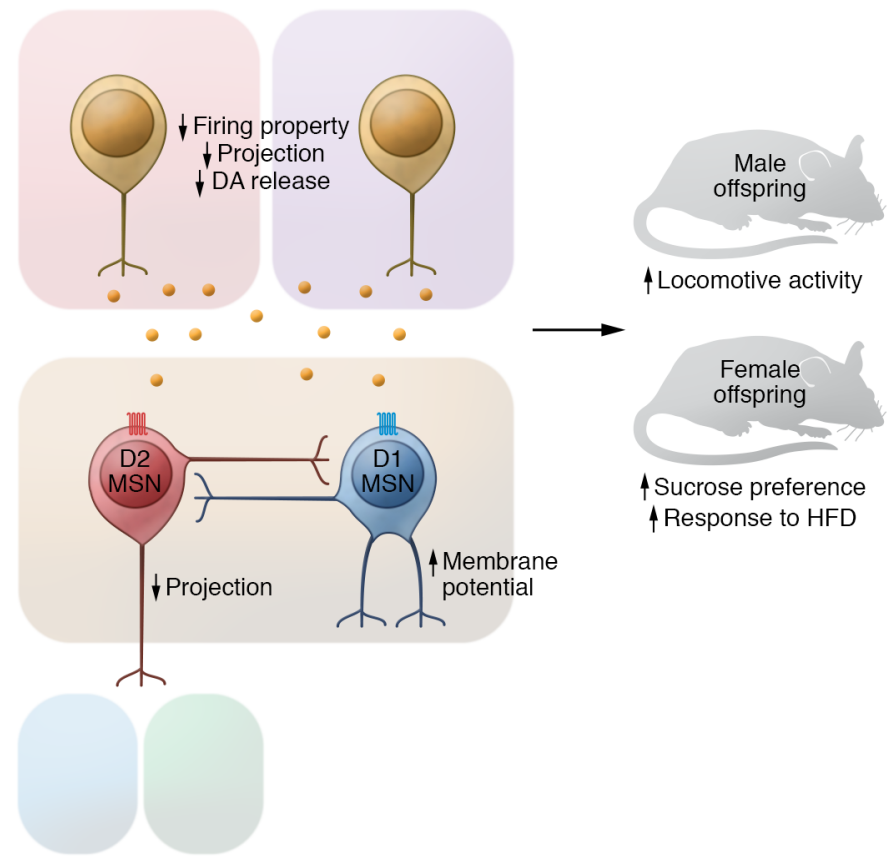

Figure 1. HFD induces alterations in the midbrain dopaminergic circuitry with behavioral outcome. (A and B) Lippert et al. (9) showed that feeding maternal mice an HFD during lactation reduced DA midbrain neuronal activity and altered offspring behavior. D1 MSNs showed increased activity, while D2 MSNs displayed decreased neuronal projections in the GP and VP.

(13). These DRs form two distinct populations, DRD1 and DRD2 MSNs in striatum and NAc, and receive input from the DA neurons of the midbrain (13). Finally, Lippert et al. assessed the electrophysiological properties of D1 MSNs of striatum and the downstream circuitry of D1 and D2 MSNs. Consistent with increased locomotion in response to novelty, the excitability of D1 MSNs increased in the dorsal striatum of male offspring from mothers exposed to HFD. Moreover, D2 MSNs displayed a robust decrease in neuronal projections in the downstream globus pallidus (GP) and ventral pallidum (VP) as well as an increased rate of spontaneous activity and response to a stimulus in the presence of decreased D2 MSN projections (ref. 9 and Figure 1).

Although Lippert et al. (9) revealed long-lasting effects on the DA circuity in the offspring of mothers exposed to HFD during lactation, the mechanisms by which nutrients and/or lipid species specifically regulate this DA circuitry remain unknown. Triglyceride (TG) and longchain fatty acid are elevated in obesity (14). Two interesting papers published recently answered some of these questions. Berland et al. showed that dietary TG can be metabolized within the MCL system and can modulate DA-associated behaviors by gating the activity of D2 MSNs through a mechanism that involves the action of the lipoprotein lipase (15). Ducrocq et al. found that mice deficient in $n-3$ polyunsaturated fatty acid (PUFA) during development resulted in motivational deficits at adulthood by increasing the inhibition of D2 MSNs onto D1 MSNs in NAc (16). Thus, TG and/or imbalance of n-3/n-6 PUFA may mediate reward-related behaviors of offspring whose mothers were exposed to HFD during pregnancy. Moreover, it was shown that the hypothalamic melanocortin circuitry has an organizational role in the development of midbrain DA system excitability during lactation (17). Whether the melanocortin system is linked to the DA circuitry described by Lippert et al. (9) has yet to be determined.

\section{Acknowledgments}

YY was supported by the Cell Science Research Foundation Researcher Training Fellowship, the Uehara Memorial Overseas Research Fellowship, and the Japan Society for the promotion of Science (JSPS) Overseas Research Fellowship (2019-2021).
Address correspondence to: Tamas L. Horvath, 310 Cedar Street, BML340, New Haven, Connecticut 06520, USA. Phone: 203.785.2525; Email: tamas.horvath@ yale.edu.

1. Jazwiec PA, Sloboda DM. Nutritional adversity, sex and reproduction: 30 years of $\mathrm{DOHaD}$ and what have we learned? J Endocrinol. 2019;242(1):T51-T68.

2. Edlow AG. Maternal obesity and neurodevelopmental and psychiatric disorders in offspring. Prenat Diagn . 2017;37(1):95-110.

3. Wang Y, Tang S, Xu S, Weng S, Liu Z. Maternal body mass index and risk of autism spectrum disorders in offspring: a meta-analysis. Sci Rep. 2016;6:34248.

4. Vogt MC, et al. Neonatal insulin action impairs hypothalamic neurocircuit formation in response to maternal high-fat feeding. Cell. 2014;156(3):495-509.

5. Abizaid A, et al. Ghrelin modulates the activity and synaptic input organization of midbrain dopamine neurons while promoting appetite. J Clin Invest. 2006;116(12):3229-3239.

6. Vucetic Z, Reyes TM. Central dopaminergic circuitry controlling food intake and reward: implications for the regulation of obesity. Wiley Interdiscip Rev Syst Biol Med. 2010;2(5):577-593.

7. Naef L, Srivastava L, Gratton A, Hendrickson $\mathrm{H}$, Owens SM, Walker CD. Maternal high fat diet during the perinatal period alters mesocorticolimbic dopamine in the adult rat offspring: reduction in the behavioral responses to repeated amphetamine administration. Psychopharma- 
$\operatorname{cology}($ Berl). 2008;197(1):83-94.

8. Rivera HM, Christiansen KJ, Sullivan EL. The role of maternal obesity in the risk of neuropsychiatric disorders. Front Neurosci. 2015;9:194.

9. Lippert RN, et al. Maternal high-fat diet during lactation reprograms the dopaminergic circuitry in mice. J Clin Invest. 2020;130(7):3761-3776.

10. Knuesel I, et al. Maternal immune activation and abnormal brain development across CNS disorders. Nat Rev Neurol. 2014;10(11):643-660.

11. Zeltser LM. Feeding circuit development and early-life influences on future feeding behaviour. Nat Rev Neurosci. 2018;19(5):302-316.

12. GBD 2015 Obesity Collaborators, et al. Health effects of overweight and obesity in 195 countries over 25 years. N Engl J Med. 2017;377(1):13-27.

13. Baik JH. Dopamine signaling in reward-related behaviors. Front Neural Circuits. 2013;7:152.

14. Cansell C, et al. Dietary triglycerides act on mesolimbic structures to regulate the rewarding and motivational aspects of feeding. Mol Psychiatry. 2014;19(10):1095-1105.
15. Berland C, et al. Circulating triglycerides gate dopamine-associated behaviors through DRD2-expressing neurons. Cell Metab. 2020;31(4):773-790.e11.

16. Ducrocq F, et al. Causal link between n-3 polyunsaturated fatty acid deficiency and motivation deficits. Cell Metab. 2020;31(4):755-772.e7.

17. Dietrich MO, et al. AgRP neurons regulate development of dopamine neuronal plasticity and nonfood-associated behaviors. Nat Neurosci. 2012;15(8):1108-1110. 